\title{
Inter-regional brain communication and its disturbance in autism
}

\section{Sarah E. SchipuI*, Timothy A. Keller and Marcel Adam Just}

Center for Cognitive Brain Imaging, Department of Psychology, Carnegie Mellon University, Pittsburgh, PA, USA

\section{Edited by:}

Barry Horwitz, National Institutes of

Health, USA

Reviewed by:

Susan Whitfield-Gabrieli,

Massachusetts Institute of Technology,

USA

John Suckling, University of

Cambridge, UK

\section{*Correspondence:}

Sarah E. Schipul, Center for Cognitive

Brain Imaging, Department of

Psychology, Carnegie Mellon

University, Pittsburgh, PA 15213, USA.

e-mail:schipul@cmu.edu
In this review article, we summarize recent progress toward understanding disturbances in functional and anatomical brain connectivity in autism. Autism is a neurodevelopmental disorder affecting language, social interaction, and repetitive behaviors. Recent studies have suggested that limitations of frontal-posterior brain connectivity in autism underlie the varied set of deficits associated with this disorder. Specifically, the underconnectivity theory of autism postulates that individuals with autism have a reduced communication bandwidth between frontal and posterior cortical areas, which constrains the psychological processes that rely on the integrated functioning of frontal and posterior brain networks. This review summarizes the recent findings of reduced frontal-posterior functional connectivity (synchronization) in autism in a wide variety of high-level tasks, focusing on data from functional magnetic resonance imaging studies. It also summarizes the findings of disordered anatomical connectivity in autism, as measured by a variety of techniques, including distribution of white matter volumes and diffusion tensor imaging. We conclude with a discussion of the implications of these findings for autism and future directions for this line of research.

Keywords: autism, brain connectivity, fMRI, DTI

\section{INTRODUCTION}

The diagnosis of autism is currently based on observed behavioral characteristics, without the aid of biological markers. The triad of behavioral impairments that characterize this neurodevelopmental disorder consists of seemingly diverse types of deficits: social reciprocity, language skills, and restricted repetitive and stereotyped patterns of behavior (American Psychiatric Association [APA], 2000). Individuals with autism also often show intact (if not enhanced) perceptual functioning (Mottron et al., 2006). Autism is a spectrum disorder, such that individuals who meet the diagnosis can have a wide range of severity of impairment in these three areas (Frith, 1989). Language impairments can vary widely, ranging from near absence of language or gestures in cases of low-functioning autism to the near normal language in high-functioning autism, but with persistent pragmatic communication difficulties (Frith and Happé, 1994). Social impairments include a lack of seeking to share enjoyment with others, a lack of emotional reciprocity, and marked impairment in the use of non-verbal behaviors such as eye-to-eye gaze (APA, 2000). Restricted repetitive and stereotyped patterns of behavior include repetitive motor mannerisms and inflexible adherence to specific routines (APA, 2000). As awareness about autism has increased dramatically in the past decade, so has research into the behavioral and biological characteristics of autism. This diverse set of behavioral impairments in autism provides a formidable research challenge, namely, to identify a biological mechanism that can explain all of them in a unified way.

The influential theory of autism known as "weak central coherence" (Frith, 1989) aimed to explain an extended set of behavioral characteristics of autism in behavioral terms. The theory proposed that underlying the distinct behavioral impairments of autism is a general deficit in the ability to integrate multiple information sources into a unified whole. This hypothesized integration deficit thus provided an account that can be applied to all three areas of impairments in autism. For example, impairments in social interactions can be attributed by weak central coherence theory to inadequate integration of many types of concurrent social information, such as the content and context of the dialog, the speaker's tone of voice, the speaker's facial expression and body language, the speaker's relationship with the listener, etc. Moreover, the sparing of certain behaviors can be explained in terms of the spared domains relying less on multiple information sources. Although the weak central coherence theory provides a compelling conceptual description that explains the clinical symptoms of autism, it does not indicate what specific cognitive or biological mechanism may underlie this general integration deficit. Several other cognitive-level theories of autism have also been proposed, including Theory of Mind disruption (Baron-Cohen et al., 1985), executive processing dysfunction (Ozonoff et al., 1991), complex information processing disorder (Minshew et al., 1997), and enhanced perceptual functioning (Mottron and Burack, 2001). While each of these theories accounts for some aspects of the behavioral symptoms of autism, neuroimaging research has the potential to identify a biological mechanism underlying and explaining the behavioral manifestations of this disorder in a unified account.

Since the advent of neuroimaging, autism researchers have attempted to use imaging methods to identify atypical characteristics of brain function and brain structure in autism. Magnetic resonance imaging (MRI) studies can be used to measure the size and properties of various gray and white matter structures in the brain. Functional MRI (fMRI) studies measure the brain activation in gray matter regions during the performance of various tasks. Furthermore, the synchronization of the activation between brain 
regions has been used as a measure of inter-regional coordination or functional connectivity. Recently, new techniques have been developed to measure the integrity of white matter pathways in the brain, providing a more precise characterization of anatomical connectivity. These neuroimaging techniques have explored differences between individuals with autism and neurotypical control individuals to investigate the possibility of localized deficits in specific areas of the brain in autism as well as network-wide disruptions throughout the brain.

What a number of fMRI studies examining brain activation in a diverse set of tasks collectively show is that the diverse behavioral symptoms of autism are not underpinned by the activity of any single brain area. Instead, these studies indicate that many different sets of brain areas are involved in the performance of tasks that show behavioral abnormalities in autism (see Di Martino et al., 2009 for a recent meta-analysis). This outcome suggests that the disturbance underlying autism is more likely a non-localized disruption. Recent neuroimaging findings have led to the hypothesis that a network-wide disruption in brain connectivity may explain the varied set of behavioral impairments in autism.

Based on neuroimaging evidence of anatomical and functional connectivity disruptions in autism, Just et al. (2004, 2007) proposed the underconnectivity theory of autism. This theory suggests that the behavioral markers of autism are directly or indirectly caused by limitations of the communication between frontal and posterior brain regions, and predicts that these limitations will impact those tasks that require extensive coordinated functioning of frontal and posterior processing centers. For example, in both language comprehension and social interaction processes, fMRI studies have shown that extensive, coordinated activity between frontal and posterior brain areas is involved. The theory accounts for restricted repetitive and stereotyped patterns of behavior in terms of the inability of the frontal executive system to exert control over posterior processing centers. The theory characterizes spared behaviors as those that do not require extensive frontal-posterior coordination, such as some perceptual processes. Thus the theory posits a biological mechanism, frontal-posterior underconnectivity, which may be able to explain the full set of diverse impairments that characterize autism.

Neuroimaging studies have demonstrated that coordinated functioning of frontal and posterior processing centers is critical for the types of behavior in which individuals with autism are impaired. Language comprehension and production require the coordinated functioning of at least the inferior frontal gyrus (Broca's area, in the left hemisphere) and the posterior superior temporal gyrus (Wernicke's area, in the left hemisphere). Similarly, social processing requires the coordinated functioning of at least medial frontal areas and posterior (right-hemisphere dominant) areas, such as the temporo-parietal junction (associated with Theory of Mind processing), the superior temporal sulcus (associated with processing biological motion), and the fusiform gyrus (associated with face processing). Restricted repetitive and stereotyped patterns of behavior may arise as a result of poor coordination between frontal executive systems that guide attention through suppression or focus and posterior systems that execute the repeated behavior. The sparing of certain visuospatial abilities in autism may be due to these processes being less reliant on the participation of frontal systems and more reliant on posterior (parietal and occipital) systems. Thus, the full set of behavioral impairments that characterize autism may be explainable by impaired integration of frontal and posterior brain systems, caused by frontal-posterior underconnectivity.

Below we review different sources of evidence of underconnectivity in autism, including measures of both functional [positron emission tomography (PET), fMRI, and electroencephalography (EEG)] and anatomical connectivity (T1-weighted structural imaging, DTI, and histological analyses). Importantly, as pointed out by Horwitz (2003), we note that methodological factors in measuring connectivity affect the inferences that can be drawn, and that because of this, the conclusions may differ depending on the particular way that connectivity is measured. Most neuroimaging studies only include high-functioning individuals (IQs in the normal range), and the majority of the studies reported here include only high-functioning individuals with autism spectrum disorders (ASD). One exception is that several structural imaging studies of children under 5 years of age do not restrict their ASD group based on IQ. We also consider the growing evidence of the relations between measures of connectivity and behavior. Finally, we discuss the implications of these findings for the treatment of autism and future directions for this area of research.

\section{FUNCTIONAL CONNECTIVITY IN AUTISM}

In a groundbreaking paper in autism neuroimaging, Horwitz et al. (1988) provided the first evidence that disrupted coordination among brain regions might be an important factor underpinning the behavioral manifestations of the disorder. This evidence was based on cross-participant, between-region correlations of regional cerebral glucose metabolism during rest, measured with PET. The striking finding from this study was that a group of adult males with autism showed a lower than normal degree of correlation between the level of metabolic activity in various activated brain regions, particularly when considering correlations between frontal and parietal regions. Interestingly, this study also found reduced acrosssubject correlations in autism for frontal-subcortical correlations of metabolic rates, but not for inter-hemispheric, homologous regions. It took over a decade for the next breakthrough to occur, a period of time during which fMRI methods were developed, which allowed for even more fine-grained, repeated measurement of task-related activation within the same participant. These new methods made it possible to extract a time series of the activation in each area and measure the correlations of the time series across areas within participants, rather than just within groups. In recent years, a variety of methods have been used to assess how functional connectivity compares between individuals with and without autism. Across many tasks and paradigms, the vast majority of studies of brain activity have reported converging findings of disrupted synchronization of brain activation in autism.

Functional connectivity is a measure of the synchronization, or covariance, of activation among different brain regions, and it is often interpreted as an indirect measure of the communication or coordination of processing between the regions. In fMRI studies, functional connectivity is typically measured by calculating the simple Pearson correlation coefficient between two time courses of activation measured in different regions. Moreover, the measurement of dyadic coordination rather than coordination 
among larger numbers of areas is simply a matter of convenience. Where appropriate, higher-order measures of coordination among a larger set of areas can be informative, showing for example, that the groupings of areas are different and smaller in autism in some tasks (Koshino et al., 2005). The more general issue concerns impaired communication between the frontal and posterior brain areas that concurrently perform a given task. If two brain areas show synchronized patterns of activation, it implies that they are performing their functions within some coordinated, coherent system, possibly involving dyadic communication and coordination between them; a better term for "functional connectivity" may have been informational connectivity. Measurements of functional connectivity can be used to examine how the communication between distinct brain areas differs between individuals with autism and neurotypical individuals.

Despite the fact that all studies of functional connectivity somehow measure inter-regional covariance of activation levels, the particular techniques used to assess this covariance can differ substantially across imaging modalities, laboratories, and studies. At the most basic level, the choice of imaging modality will determine the spatial and temporal resolution at which such covariance can be measured. As noted above, early PET studies (e.g., Horwitz et al., 1988) lacked the temporal resolution to evaluate functional connectivity within individual subjects, and later PET studies could only evaluate such connectivity at the temporal resolution of lengthy (e.g., 45-s) blocks of data acquisition (Castelli et al., 2002). With the advent of fMRI, these limitations on temporal resolution were removed, but different techniques resulted in functional connectivity being measured at different spatial resolutions.

One approach that is well-suited to fMRI measurement of the coordination of information processing involves averaging the activation time course of all the voxels in each region which have been shown to be activated in the task at hand. The analyses then examine the correlations among all pairs of these averaged time series in a given task (e.g., Just et al., 2004). Alternatively, voxel-based analyses can measure the synchronization between one seed region [based on a single voxel or an average of all voxels in a predefined region of interest (ROI)] and all the remaining voxels throughout the brain. This latter method focuses on measuring the synchronization between the activation in a specific brain structure and the rest of the brain, rather than examining connectivity between all possible pairs of regions, but allows group differences in this connectivity with a single region to be assessed in each voxel over the entire brain.

In the measurement of functional connectivity in fMRI, it is important to note which range of frequencies is being included in the measurement and with what rationale. The majority of fMRI studies of functional connectivity correlate activation measurements that occur once every second or $2 \mathrm{~s}$ during task performance. These studies focus on the synchronization of activation that is assumed to reflect conjoint modulations of the information processing activity in each of two regions. By contrast, a few fMRI studies of functional connectivity have instead examined only the slow activation changes (occurring over the course of $10 \mathrm{~s}$ or more) that are independent of task performance. (This is done by removing frequencies above $0.1 \mathrm{~Hz}$.) This approach assesses whether the functional connectivity differences in autism are present in slower-changing biological activity unrelated to cognition. This review focuses on the findings of task-dependent functional connectivity differences in autism related to cognitive processing, but also includes the findings of task-independent functional connectivity.

\section{FRONTAL-POSTERIOR FUNCTIONAL CONNECTIVITY IN AUTISM}

The most consistent finding of functional connectivity differences in autism is a pattern of lower frontal-posterior functional connectivity relative to neurotypical individuals. Many studies have found decreased synchronization between whatever frontal and posterior regions are recruited for a given task. For example, in a task designed to require the integration of spatial processing and language comprehension, decreased functional connectivity was found in autism ${ }^{1}$ between frontal language regions and parietal spatial processing regions (Kana et al., 2006). Participants had to judge sentences such as the following as true or false: The number 8 when rotated $90^{\circ}$ looks like a pair of eyeglasses. Similarly, in a working memory task where participants were asked to judge whether a face they were viewing was the same as one seen previously, decreased functional connectivity was found in autism between frontal executive regions and the fusiform gyrus, an area involved in face processing (Koshino et al., 2008). This latter study illustrates that what is impaired in autism is not just the functioning of a particular brain area (although the activation in the fusiform gyrus was in a slightly offset location in autism), but that, in addition, the functional connectivity between the fusiform gyrus and frontal regions was abnormally low. Furthermore, this pair of studies illustrates the point that functional underconnectivity emerges between whatever frontal and posterior regions are centrally involved in the task. Almost all complex language, social, and executive tasks, precisely where behavioral deficits are typically found in autism, would be expected to show frontal-posterior functional underconnectivity. (An example of a task that might be expected not to draw heavily on frontal regions is a perceptual task that can be performed without strategic control, and this is also the type of task where one might expect sparing in autism. It will be interesting to learn about the functional connectivities among posterior regions in autism in such tasks, which have not yet been reported).

Similar findings of lower functional connectivity in autism in relevant frontal and posterior areas have been reported in a wide variety of cognitive tasks. For example, in a passage comprehension task in which participants had to make inferences about the characters' intentions, decreased functional connectivity in autism was found between frontal and parietal Theory of Mind areas, as well as between frontal language areas and parietal Theory of Mind areas (Mason et al., 2008). Figure 1 depicts the frontal-posterior underconnectivity in autism during this task (where line width corresponds to the group difference in functional connectivity). In a Tower of London task, decreased functional connectivity in autism was found between frontal and parietal working memory areas (Just et al., 2007). In a task which required participants to make inferences about the intentions of computer-animated geometric

${ }^{1}$ As we report findings, we use either autism or autism spectrum disorders (ASD) to reflect the author's use. The two terms generally have different qualifications, with ASD typically being a superordinate category that may include autism, Asperger's syndrome, and pervasive developmental disorder. 


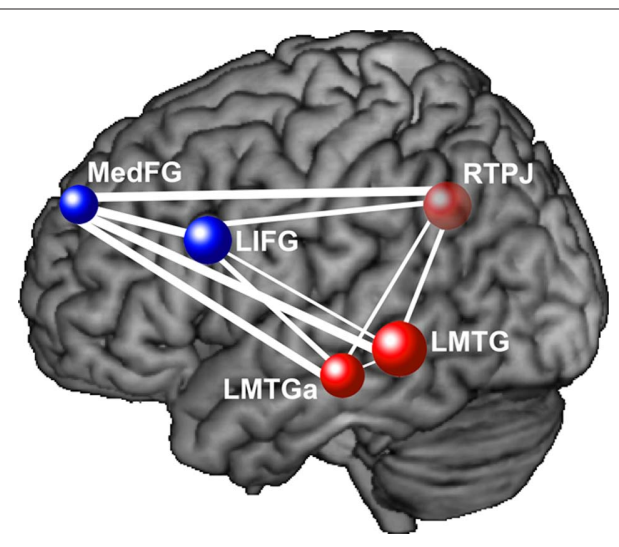

FIGURE 1 |This diagram depicts functional underconnectivity, specifically between frontal and posterior areas, in autism during an inferential text comprehension task. The width of each connecting line represents the $t$-value of the difference in functional connectivity between the participants with autism and the neurotypical participants. Blue nodes are frontal regions and red nodes are posterior regions. The widest lines (reflecting the greatest group differences) are those connecting frontal and posterior regions. Data from Mason et al. (2008), with permission. MedFG, medial frontal gyrus; LIFG, left inferior frontal gyrus; RTPJ, right temporoparietal junction; LMTG, left middle temporal gyrus; LMTGa, anterior left middle temporal gyrus.

figures, individuals with autism had lower functional connectivity between frontal and posterior Theory of Mind areas (Kana et al., 2009). In a reading comprehension task with sentences of varying complexity, underconnectivity was found in autism between frontal and posterior areas involved in language comprehension and working memory (Just et al., 2004). In a working memory task with alphabetic characters, lower functional connectivity was reported between frontal and parietal working memory areas (Koshino et al., 2005). In a complex inhibition task, functional connectivity was lower in autism between the frontal inhibition network and the inferior parietal lobe (Kana et al., 2007). In a cognitive control task, lower functional connectivity was reported in ASD between the frontal executive system and several posterior regions in the brain, including parietal working memory areas and the visual cortex (Solomon et al., 2009). In the context of a working memory task with faces and houses (similar to that of Koshino et al., 2008, described above), lowered functional connectivity was found in ASD between the fusiform gyrus and the amygdala (both related to face processing), as well as between the fusiform gyrus and the posterior cingulate (Kleinhans et al., 2008). These studies collectively illustrate that functional underconnectivity has been observed in autism in a wide variety of frontal-posterior pairs. The communication between the members of each of these pairs is necessary for the integration of the multiple cognitive processes required for a given task. These findings support the underconnectivity theory's claim that decreased frontal-posterior connectivity in autism specifically affects behaviors that require the extensive coordinated functioning of frontal and posterior processing centers.

Findings of underconnectivity in autism have also been reported in the absence of task performance, while participants are at rest. Such "resting state" studies offer the advantage of measuring the functional connectivity between different brain regions in spontaneous, naturally occurring patterns of brain activity. Studies of resting state in autism have revealed a "default network" of areas (very similar to the neurotypical default network), which includes the medial prefrontal cortex, anterior and posterior cingulate cortex, the precuneus, and the inferior parietal cortex (Cherkassky et al., 2006). The majority of resting state studies have found decreased functional connectivity in autism in both frontal-posterior pairs and other pairs. Decreased functional connectivity in autism during rest was found in $94 \%$ of possible pairs among the default network ROIs, including between the anterior cingulate and the posterior cingulate and precuneus (Cherkassky et al., 2006). In another study, decreased functional connectivity in ASD was found within the task-negative network (medial prefrontal cortex, posterior cingulate, and angular gyrus), but not in the task-positive network (intraparietal sulcus, superior precentral sulcus, and middle temporal gyrus; Kennedy and Courchesne, 2008). Functional connectivity was also found to be lower in ASD between the superior frontal gyrus and the posterior cingulate (Monk et al., 2009). Notably, Monk et al. (2009) found that among posterior regions (involving posterior cingulate, temporal lobe, and parahippocampal gyrus), the functional connectivity was higher in ASD, providing a suggestion that connectivity among posterior regions may not only be unaffected in autism, but that there may be compensatory posterior overconnectivity. Decreased functional connectivity was also found in adolescents with ASD between the posterior cingulate and 9 of the 11 other default network regions, including medial prefrontal and superior frontal (Weng et al., 2010). These studies show decreased functional connectivity within the default network in autism while participants are at rest, suggesting that functional underconnectivity in autism is not necessarily dependent on the performance of overt complex cognitive tasks.

As mentioned above, task-independent functional connectivity has also been examined in autism. This method focuses on the synchronization of the task-independent, very low-frequency activation fluctuations that are not related to cognitive processing. It is unclear how this task-independent measure of synchronization is related to disruption of thought in autism. Nevertheless, several such studies have also found functional connectivity differences in autism between frontal and posterior areas. Functional underconnectivity in autism has been reported between the visual cortex and several frontal regions (Villalobos et al., 2005), and lower frontalposterior connectivity was found in ASD both with and without regressing out task effects in an overt verbal fluency task (Jones et al., 2010). However, there have also been findings of increased frontal-posterior functional connectivity in ASD using this method (Noonan et al., 2009). The findings of atypical task-independent functional connectivity in autism may suggest that even at the biological level, differences are present in this disorder.

\section{FUNCTIONAL CONNECTIVITY IN AUTISM IN PAIRS OTHER THAN FRONTAL-POSTERIOR}

Disturbances of functional connectivity in autism have also been reported between pairs of regions other than frontal-posterior pairs, but the findings in such pairs have been less consistent across studies, as shown in Table 1. For example, lower functional connectivity in ASD has been reported between the amygdala and temporal and frontal regions (Monk et al., 2010), between the anterior cingulate 
Table 1 | Summary of fMRI functional connectivity results.

\begin{tabular}{|c|c|c|}
\hline Task & $\begin{array}{l}\text { Pairs of } \\
\text { regions }\end{array}$ & $\begin{array}{l}\text { Functional } \\
\text { underconnectivity in autism }\end{array}$ \\
\hline $\begin{array}{l}\text { Task } \\
\text { performance }\end{array}$ & $\begin{array}{l}\text { Frontal- } \\
\text { posterior pairs }\end{array}$ & $\begin{array}{l}\text { Just et al. (2004, 2007), Koshino } \\
\text { et al. (2005, 2008), Kana et al. } \\
\text { (2006, 2007, 2009), Kleinhans et al. (2008), } \\
\text { Mason et al. (2008), Solomon et al. (2009) }\end{array}$ \\
\hline $\begin{array}{l}\text { Task } \\
\text { performance }\end{array}$ & Other pairs & $\begin{array}{l}\text { Kleinhans et al. (2008), Mostofsky } \\
\text { et al. (2009), Agam et al. (2010), } \\
\text { Lombardo et al. (2010), Monk et al. (2010) }\end{array}$ \\
\hline Resting state & $\begin{array}{l}\text { Frontal- } \\
\text { posterior pairs }\end{array}$ & $\begin{array}{l}\text { Cherkassky et al. (2006), Kennedy } \\
\text { and Courchesne (2008), Monk } \\
\text { et al. (2009), Weng et al. (2010) }\end{array}$ \\
\hline
\end{tabular}

and frontal eye fields (Agam et al., 2010), within a motor network consisting of primary and supplementary motor areas, anterior cerebellum, and the thalamus (Mostofsky et al., 2009), between the prefrontal cortex and premotor and somatosensory cortices (Lombardo et al., 2010), and between the fusiform gyrus and the amygdala, the posterior cingulate and the cuneus (Kleinhans et al., 2008). Based on activation results, Silk et al. (2006) proposed a disruption in the frontostriatal network in ASD. While these studies all report functional underconnectivity in autism in non-frontalposterior pairs, the findings vary across a wide variety of pairs of regions and across a large range of tasks, making it difficult to isolate a specific pattern of disturbance. Furthermore, the fact that two studies have reported increased functional connectivity in ASD in non-frontal-posterior pairs of areas (Welchew et al., 2005; Monk et al., 2010) further illustrates the variability of functional connectivity findings in autism for pairs of regions other than frontal-posterior pairs.

Functional MRI studies of task-independent functional connectivity have also found group differences in other pairs of areas, often focusing on subcortical regions. Although decreased functional connectivity in autism was reported between the visual cortex and the thalamus and cerebellum (Villalobos et al., 2005), and between the superior frontal gyrus and the caudate (Turner et al., 2006), studies have also found increased connectivity in autism in many pairs involving subcortical regions. Increased functional connectivity in autism was reported between the thalamus and several areas throughout the cortex (Mizuno et al., 2006), as well as between the caudate and many other regions, including frontal regions, the cingulate, and the cuneus (Turner et al., 2006). Increased taskindependent functional connectivity in ASD was also reported in frontal-frontal and posterior-posterior pairs (Noonan et al., 2009). The most consistent finding of increased task-independent functional connectivity in autism is in subcortico-cortical pairs. Because this method measures task-independent synchronization, it remains unclear how these findings may be related to the integration of cognitive processes during task performance, although they may suggest differences in autism even at the biological level.

As this section has illustrated, functional connectivity differences in autism have also been found in pairs of areas that are not frontal-posterior, in both task-dependent and task-independent measures. While the majority of studies report lower functional connectivity in autism in these other pairs, some studies have reported increased functional connectivity in autism, most often in subcortico-cortical pairs. It should be noted that all findings of overconnectivity in ASD come from studies using non-standard methods, such as psychophysiological interaction connectivity analysis (Monk et al., 2010), multidimensional scaling (Welchew et al., 2005), and task-independent functional connectivity analyses (Mizuno et al., 2006; Turner et al., 2006; Noonan et al., 2009), and, with the exception of Noonan et al. (2009), all of these studies reported differences in pairs involving the amygdala or subcortical structures. Further research is necessary to determine if these findings of increased functional connectivity in autism reflect unique properties of subcortico-cortical connections in autism. In addition, several studies reported decreased functional connectivity in autism in non-frontal-posterior pairs, but this varied set of findings has yet to illustrate a clear pattern of disturbance. Future research in this area should be able to produce a more complete account of functional connectivity disturbances in autism, using a variety of tasks and measurement methods. It is possible that whatever biological mechanisms lead to frontal-posterior underconnectivity in autism may have also affected other connections, but to a lesser degree or less consistently. It may be that frontal-posterior connections are most strongly affected by the biological disturbance underlying autism, but functional connectivity differences may occasionally appear in any number of other connections.

\section{ALTERNATIVE METHODS OF ASSESSING FUNCTIONAL CONNECTIVITY}

Functional connectivity in fMRI data has also been measured with various other techniques. For example, independent component analysis (ICA) identifies temporally coherent networks by selecting spatially independent brain areas whose hemodynamic time courses closely co-vary. ICA is a data-driven method which does not rely on a priori ROIs or the performance of a cognitive task. An ICA examination of resting state fMRI data revealed that individuals with ASD had decreased strength of functional connectivity between both the precuneus and the anterior cingulate cortex and a default network consisting of the posterior cingulate cortex, the inferior parietal lobule, and the medial prefrontal cortex (Assaf et al., 2010). Thus, ICA is a novel technique which is producing findings in accordance with previous reports of functional underconnectivity in ASD during resting state (Cherkassky et al., 2006; Kennedy and Courchesne, 2008; Weng et al., 2010).

Another data-driven approach to measuring functional connectivity is the measurement of regional homogeneity ( $\mathrm{ReHo}$ ), which tests for local correlations in fMRI time series. This technique calculates the Kendall's coefficient of concordance (KCC) for each voxel with its neighboring voxels, thereby estimating the local connectivity of every voxel in the brain. While the two existing studies of ReHo in ASD have somewhat divergent results, both find decreased ReHo in young adolescents with ASD in the frontal lobe (Paakki et al., 2010; Shukla et al., 2010). However, while Shukla et al. (2010) also found decreased ReHo in the superior parietal lobule and increased ReHo in the temporal lobe, Paakki et al. (2010) instead found decreased ReHo in right superior temporal sulcus and cerebellum with increased ReHo in the right thalamus, left inferior frontal gyrus/subcallosal gyrus, and cerebellum. These divergent results may stem from methodological differences: 
Shukla et al. (2010) calculated ReHo as the KCC of each voxel and its six neighboring voxels, while Paakki et al. (2010) used 27 neighboring voxels. Thus, the two studies may be examining different levels of neuronal organization. Nevertheless, this method may provide new insight into local functional connectivity in ASD, although it is at yet unclear how this measure would relate to longdistance inter-regional functional connectivity. Graph theory is another tool now being used to characterize both functional and anatomical networks in the neurotypical brain (see Bullmore and Sporns, 2009, for a review), and future research may apply these techniques to the investigation of underconnectivity in autism. Many novel techniques are being used to examine characteristics of brain connectivity and have the potential to further illuminate the characteristic properties of the brains of individuals with autism.

Yet another measure that has also shown differences in ASD is effective connectivity (Wicker et al., 2008), a measure that attempts to assess the influence of the activation in one region on the activation in another region (Friston, 1994). Wicker et al. (2008) used structural equation modeling to assess effective connectivity in the BOLD response in a dynamic face processing task. The resulting findings of weaker path coefficients in ASD in many connections with the prefrontal cortex converge with Koshino et al.'s (2008) findings of decreased frontal-posterior functional connectivity in a working memory task with faces, discussed above. A potential contribution of effective connectivity measures seems most likely in cases where there might be a group difference in the inter-regional control relations. However, the activity among association areas may be too interactive to allow detection of differences in interregional influence.

Finally, functional connectivity differences in ASD have also been found through other imaging methods, including PET (Castelli et al., 2002), EEG (Murias et al., 2007), and magnetoencephalography (MEG; Coskun et al., 2009). While fMRI and PET measure the blood flow in the brain on the order of seconds, EEG and MEG measure electrical and magnetic signals of neuron firing on the order of milliseconds and, therefore, may reflect properties of brain function that are distinct from those revealed by fMRI data. However, EEG studies have also found reduced long-range coherence in ASD between frontal and posterior areas in both the alpha band (Murias et al., 2007) and the delta band (Barttfeld et al., 2011), as well as elevated short-range coherence in both the theta band (Murias et al., 2007) and the delta band (Barttfeld et al., 2011). Furthermore, Barttfeld et al. (2011) used graph theory metrics to determine that the network of activated brain regions is less well organized in ASD, as reflected by longer path lengths, less clustering, and increased modularity. While the focus of this review is on functional connectivity as measured in fMRI data, current work in EEG is also providing insights into the characteristics of brain connectivity in autism.

\section{ANATOMICAL CONNECTIVITY IN AUTISM}

The lower frontal-posterior functional connectivity in autism might be attributable to an impairment in anatomical connectivity. (It is also logically possible for the functional connectivity between two areas to be poor because of the communication protocols rather than the carrier anatomical link.) The anatomical evidence that is most relevant to underconnectivity is that by adulthood, individuals with autism tend to have more poorly developed white matter pathways relative to neurotypical individuals, as described below. Evidence from several neuroimaging methods has also revealed an atypical developmental pattern of cortical connectivity in autism. Individuals with autism show a large increase in white matter growth in very early childhood, followed by a period of relatively slowed growth. There are several sources of evidence of white matter abnormalities in autism that can affect connectivity.

\section{VOLUME MEASUREMENTS OF WHITE MATTER IN AUTISM}

Infants who are later diagnosed with ASD show typical brain characteristics at birth, but shortly thereafter show atypically fast brain growth. Based on measurements of head circumference (a rough estimate of brain size), newborns with ASD had slightly smaller head circumferences than typical infants, but then showed an atypically large increase in head size within the first 6-14 months of age, resulting in atypically large head circumferences (Courchesne et al., 2003). Structural MRI measurements of brain volumes have revealed converging evidence of increased volumes in children with autism between the ages of 2 and 4 years in both cerebral gray and white matter. Ninety percent of 2- to 4 -year-olds with autism had increased brain volumes in gray and white matter relative to neurotypical children (Courchesne et al., 2001). Greater brain volumes in 2- to 4-year-olds with autism were more pronounced in the frontal lobe, with no differences in the occipital lobe (Carper et al., 2002). Together, these findings suggest that children with autism show atypically rapid increases in frontal gray and white matter in the first 2 years of life.

The pattern of rapid brain growth in autism reverses around age 4 , such that children with autism then show a decreased rate of brain growth in white matter from ages 3 to 12 (Courchesne et al., 2001). Neurotypical children showed a 59\% increase in white matter volume during this time period, while children with autism showed only a $10 \%$ increase. Greater volume of white matter was found, particularly in frontal radiate white matter in 5- to 11-year-olds with autism, as well as volumetric increases and decreases in a number of other regions, indicating the disruption of the white matter (Herbert et al., 2004). The period of slowed growth eventually results in a smaller volume of white matter in adolescents with autism relative to neurotypical adolescents (Courchesne et al., 2001). A similar pattern was found in cortical gray matter. Thus, brain volume measurements have revealed that the rate of brain growth in autism slows after age 4, leading to a decreased volume of white matter in adolescents with autism relative to neurotypical adolescents. Given that white matter is the medium which is used for inter-regional brain communication, it seems incontrovertible that brain connectivity is disrupted in autism.

The presence of a temporary excess of white matter in children with autism does not necessarily denote superior connectivity. These data simply indicate that white matter in the frontal lobe is increasing in volume earlier in children with autism than in neurotypical children. However, the white matter may not be developing properly during its rapid growth in autism. Therefore, it is useful to also examine the quality of white matter in individuals with autism using diffusion tensor imaging. 


\section{DIFFUSION TENSOR IMAGING ASSESSMENT OF WHITE MATTER IN AUTISM}

Diffusion tensor imaging methods can be used to examine the integrity of white matter tracts in the brain. This technique measures the movement of water molecules as they diffuse along white matter tracts, reflecting characteristics of white matter architecture. An increasing number of studies are finding areas of lower structural integrity in autism.

Children with autism show areas of decreased white matter integrity relative to typically developing children, similar to the developmental pattern reflected in volume measurements of white matter. Fractional anisotropy (FA) is a measure of the coherence of diffusion directionality, such that lower FA suggests decreased white matter integrity. FA was found to be reduced in children and adolescents with autism in white matter adjacent to the prefrontal cortex, the anterior cingulate, and the temporo-parietal junctions (Barnea-Goraly et al., 2004). Lower FA has also been reported in adolescents with autism in frontal-temporal pathways (Sahyoun et al., 2010). Decreased white matter integrity was found in both adolescents and children with ASD in the arcuate fasciculus, which connects frontal and posterior language regions (Fletcher et al., 2010; Kumar et al., 2010). FA was lower in short- but not longrange fibers in the frontal lobe in individuals with ASD as young as 5 (Sundaram et al., 2008). Thus, many studies have shown that older children and adolescents with autism show reduced white matter integrity in the frontal lobe, relative to typically developing children.

However, several studies involving children with ASD of varying age ranges have found areas of not only decreased but also increased FA in ASD (ages 1-3 years old: Ben Bashat et al., 2007; ages 6-14 years old: Cheung et al., 2009; Ke et al., 2009; ages 10-18 years old: Cheng et al., 2010). Such heterogeneous findings might arise because of large variance due to rapid developmental changes in the integrity of white matter in autism during this period of childhood. Despite the inconsistencies of DTI findings in such young children with autism, older children and adolescents with autism consistently show decreased white matter integrity relative to their neurotypical peers.

Decreased white matter integrity in autism has been found to persist into adulthood. Several clusters of decreased FA near the corpus callosum in the frontal and temporal lobes were reported in autism participants between the ages of 10 and 35 (Keller et al., 2007). Lower FA was also reported in individuals with ASD from ages 7 to 33 in the corpus callosum (Alexander et al., 2007) and in the temporal lobe (Lee et al., 2007). Thus, decreased white matter integrity persists into adulthood, possibly constituting the biological basis of the decreased functional connectivity in adults with autism.

It is as yet undetermined how early brain overgrowth in autism may be related to decreased white matter integrity in adulthood. It is noteworthy that early brain overgrowth is greatest in the frontal lobe, while impaired functional connectivity later in life is most prevalent in synchronizations between the frontal lobe and more posterior regions. Evidence of smaller and more numerous cortical minicolumns in autism in the frontal and temporal lobes (Casanova et al., 2002) may suggest an increased formation of short-range connections within these lobes. Such increased short-range connectivity may be the cause or consequence of poor inter-regional connectivity. Future investigation of the molecular origins underlying brain connectivity differences in autism may further illuminate the connectivity phenomena.

\section{RELATING FUNCTIONAL CONNECTIVITY, ANATOMICAL CONNECTIVITY, AND BEHAVIOR IN AUTISM}

The underconnectivity theory of autism proposes that anatomical and functional connectivity are related, and that both of these measures should be related to behavior. Findings of correlations across subjects between functional connectivity, anatomical connectivity, and behavior provide support for this hypothesis. In fact, neuroimaging studies have shown evidence of such relations between each of these measures of connectivity and behavior.

\section{RELATING FUNCTIONAL CONNECTIVITY AND BEHAVIOR IN AUTISM}

If decreased functional connectivity is truly a neural mechanism underlying behavioral impairments of autism as proposed, one would expect functional connectivity measurements to be related to behavioral markers of autism. Several studies have found a relation between functional connectivity measures and measures of autism characteristics as measured by the Autism Diagnostic Observation Schedule (ADOS) and the Autism Diagnostic Interview-Revised (ADI-R). The ADOS and ADI-R are diagnostic tools that measure behavioral characteristics of autism. The ADOS has subscores for social behavior and communication, which are combined into a total score. The ADI-R has three separate scores for reciprocal social interaction, communication and language, and restricted and repetitive, stereotyped interests and behaviors. Although these measures were developed to optimize their diagnostic rather than their psychometric properties (e.g., the range of scores on these tests is fairly low among people with high-functioning autism), they nevertheless produce sensible correlations with functional and anatomical connectivity measures. One study found that high-functioning individuals with more severe cases of autism (higher total ADOS score) had lower frontal-parietal functional connectivity (Just et al., 2007). Other studies have also shown that individuals with ASD with poorer social functioning (high ADI-R social score) have lower functional connectivity between the superior frontal gyrus and posterior cingulate (Monk et al., 2009; Weng et al., 2010). Lower functional connectivity between frontal structures and the posterior cingulate was found in participants with more severe repetitive behaviors (Weng et al., 2010). These findings suggest that impaired frontal-posterior functional connectivity may be directly related to more severe autistic traits in language, social functioning, and repetitive behaviors.

There have also been reports of increased functional connectivity in non-frontal-posterior pairs in individuals with more severe autistic traits. The higher functional connectivity between posterior regions may develop in some people with autism in compensation for decreased connectivity between frontal and posterior areas, with more such compensation in cases with poorer frontal-posterior connectivity. Adolescents with ASD who had higher functional connectivities within various areas of the default network during rest had lower abilities in both verbal and non-verbal communication (Weng et al., 2010). Higher functional connectivity between the posterior cingulate and the parahippocampal gyrus was associated with more severe repetitive behaviors (Monk et al., 2009), as was higher functional connectivity between the anterior cingulate and the frontal eye fields (Agam et al., 2010).

Individuals with autism with the most impaired inter-regional communication between frontal and posterior areas also show larger behavioral deficits. Thus, if an intervention were able to facilitate the 
communication between frontal and posterior processing centers in individuals with autism, this may result in improvements in the behavioral impairments associated with autism.

\section{RELATING ANATOMICAL CONNECTIVITY AND BEHAVIOR IN AUTISM}

Studies have also found relations between anatomical connectivity and behavioral markers of autism. Lower FA (lower white matter integrity) was related to more severe disruptions manifested in social function, communication, and repetitive behaviors (as measured by ADI-R scores; Cheung et al., 2009). This finding suggests that decreased anatomical connectivity may lead to greater behavioral impairments in autism. Specifically, decreased integrity in fronto-striato-temporal pathways was related to more impaired social functioning and communication abilities, while decreased integrity in more anterior and posterior pathways, including the splenium of the corpus callosum, was related to more severe repetitive behaviors. Lower FA (specifically in the white matter underlying the anterior cingulate cortex) was also associated with more severe ADI-R repetitive behavior scores (Thakkar et al., 2008). The majority of findings have shown that decreased integrity in white matter tracts is associated with more severe autistic behaviors, suggesting that impaired anatomical connectivity may underlie the behavioral characteristics of autism.

\section{RELATING FUNCTIONAL AND ANATOMICAL CONNECTIVITY IN AUTISM}

Several studies have reported correlations between functional connectivity and corpus callosum area measurements in autism. The corpus callosum is a major white matter tract connecting the two hemispheres, and many studies have reported smaller corpus callosum sizes in ASD (Vidal et al., 2006; Hardan et al., 2009; Keary et al., 2009). Although this effect has not always been found at lower magnetic field strengths (0.5-1.5 T: Gaffney et al., 1987; Rice et al., 2005; Tepest et al., 2010), two meta-analyses found that smaller corpus callosum size in ASD is significant across MRI studies (Stanfield et al., 2008; Frazier and Hardan, 2009). Furthermore, magnetic field strength was shown to be a marginally significant predictor of group differences, such that stronger magnets show larger discrepancies in corpus callosum size between ASD and neurotypical participants (Frazier and Hardan, 2009). Because the corpus callosum is such a critical pathway in the brain and is often found to be smaller in autism, corpus callosum size is sometimes used as an index of general anatomical connectivity in the brain. Several studies have found measures of functional connectivity to be positively correlated with corpus callosum size in autism (Cherkassky et al., 2006; Kana et al., 2006; Just et al., 2007; Mason et al., 2008), as illustrated in Figure 2. Furthermore, in all of the above studies, this correlation was not found in the control group, suggesting that only in autism is communication between distinct brain areas constrained by impaired anatomical connectivity.

\section{IMPLICATIONS FOR THE TREATMENT OF AUTISM}

The body of work described in this article provides substantial evidence that behavioral impairments in autism may be caused by limitations in brain connectivity. These findings suggest that it may be fruitful to develop intervention methods that aim to improve inter-regional communication in the brain in individuals with autism.

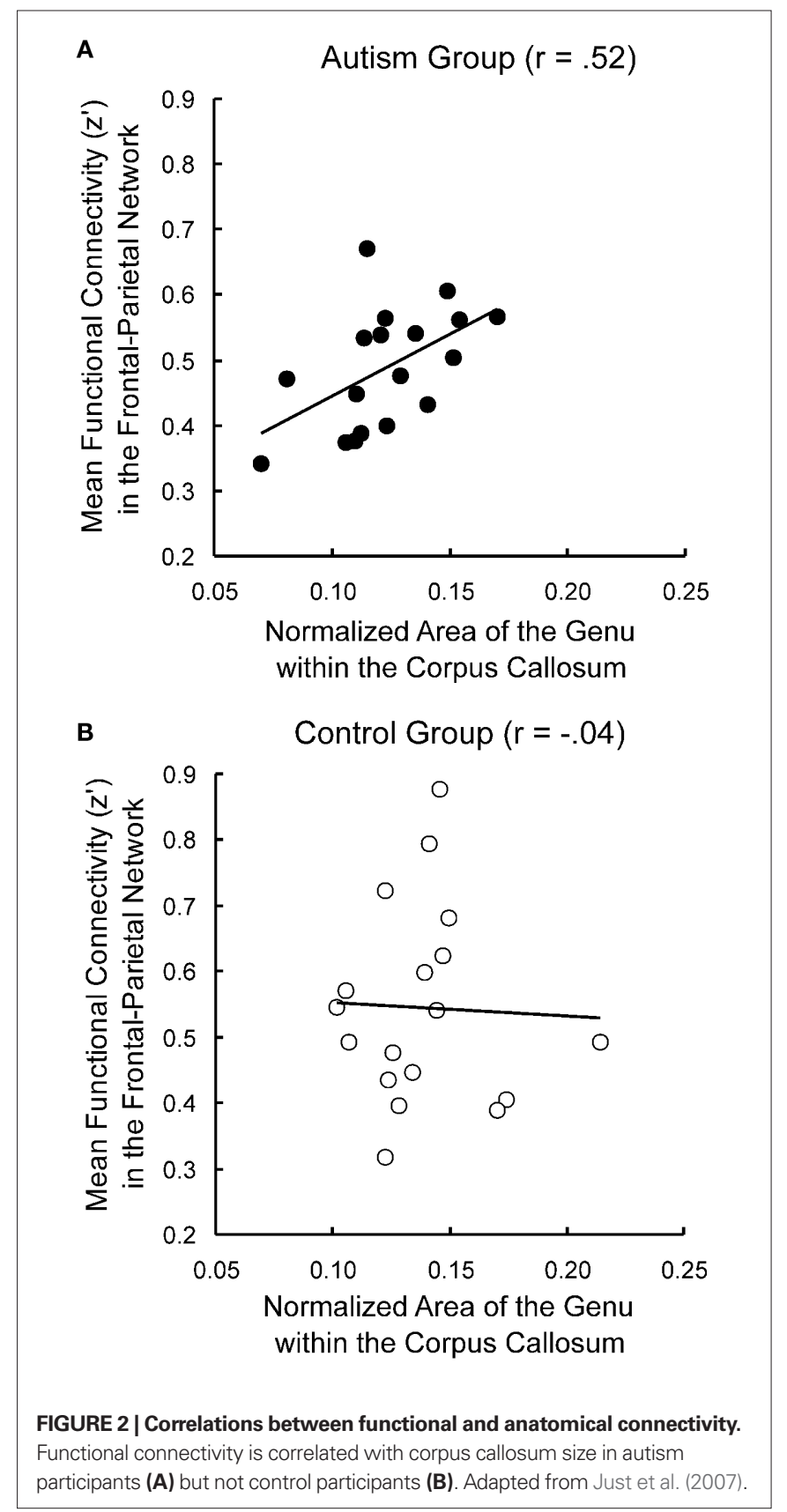

Functional MRI evidence has shown that it is possible to observe increases in functional connectivity through learning over a very short time period in neurotypical participants (Büchel et al., 1999). Schipul et al. (2008) examined functional connectivity during learning in autism: participants both with and without autism learned to discriminate between lying and truth-telling avatars (animations of human-like speakers) over the course of a 20-min experiment. The results showed that the functional connectivity increased in both the autism and control groups as they learned to perform the task, but the control participants showed a much larger increase in functional connectivity than the autism participants throughout the brain. These findings suggest that as participants practice a task and learn new strategies, inter-regional communication may increase, perhaps 
contributing to improved behavioral performance. Therefore, autism intervention methods that incorporate guided repetition of tasks involving frontal-posterior coordination may be able to temporarily or permanently improve inter-regional brain communication.

White matter connectivity has been shown to be amenable to such intervention. A study of children with impaired reading ability found that after $100 \mathrm{~h}$ of intensive remedial instruction in reading, the structural integrity increased in the specific white matter structure that was previously impaired in these children (Keller and Just, 2009). Moreover, the degree of white matter improvement was correlated with the degree of reading improvement. This finding suggests that it is possible to improve the wiring of the brain through behavioral training. Therefore, it may be possible to design intervention methods for autism which will improve the anatomical connectivity and inter-regional communication in the brain, which may lead to improvements in behaviors that are often impaired in autism.

\section{RELATION TO OTHER DISORDERS}

Altered functional connectivity has also been found in other disorders, including schizophrenia (Meyer-Lindenberg et al., 2001), attention deficit hyperactivity disorder (Tian et al., 2006), multiple sclerosis (Au Duong et al., 2005), and dyslexia (Pugh et al., 2000). These findings suggest that disordered brain connectivity may underlie a variety of cognitive impairments. While autism is primarily associated with frontal-posterior underconnectivity, preliminary evidence suggests that these other disorders are linked with impairments in other types of connections (Pugh et al., 2000; Meyer-Lindenberg et al., 2001; Au Duong et al., 2005; Tian et al., 2006). Therefore, the location of the impaired connections may be specific to the associated cognitive impairments. For example, dyslexia, which impairs reading, has been associated with underconnectivity between the angular gyrus, an area implicated in reading, and occipital and temporal regions (Pugh et al., 2000). Furthermore, the disordered functional connectivity associated with each of these disorders may arise for different reasons and may be either a cause or a consequence of the disorder. Moreover, the disordered functional connectivity can arise at different times in development: whereas disordered brain connectivity appears in childhood in autism, it may not arise until later in disorders such as schizophrenia, whose symptoms first appear at a much later age than autism. The impairments associated with the various psychiatric disorders include hallucinations, disorganized speech, attention problems, hyperactivity, and reading difficulties, illustrating the wide range of effects that may be associated with disordered brain connectivity. Thus, assessments of functional and anatomical connectivity may be able to provide useful insights into a number

\section{REFERENCES}

Agam, Y., Joseph, R. M., Barton, J. J., and Manoach, D.S. (2010). Reduced cognitive control of response inhibition by the anterior cingulate cortex in autism spectrum disorders. Neuroimage 52, 336-347.

Alexander, A. L., Lee, J. E., Lazar, M., Boudos, R., DuBray, M. B., Oakes, T. R., Miller, J. N., Lu, J., Jeong, E. K., McMahon, W. M., Bigler, E. D., and Lainhart, J.E. (2007). Diffusion tensor imaging of the corpus callosum in autism. Neuroimage 34, 61-73.

American Psychiatric Association. (2000). Diagnostic and Statistical Manual of Mental Disorders, 4th Edn. Washington, DC: American Psychiatric Association.

Assaf, M., Jagannathan, K., Calhoun, V. D., Miller, L., Stevens, M. C., Sahl, R., O’Boyle, J. G., Schultz, R. T., and Pearlson, G. D. (2010). Abnormal functional connectivity of default

of neurological and psychiatric illnesses, but the basis of any connectivity disorder may vary from illness to illness. But regardless of its basis, disordered brain connectivity can give rise to a wide variety of behavioral impairments, indicating the centrality of brain connectivity to all types of cognition.

\section{FUTURE DIRECTIONS}

The majority of the functional connectivity studies reviewed above involved adult participants with high-functioning autism. In order to fully explain this disorder, especially in light of the anatomical findings of the atypical development of white matter, it is necessary to examine functional connectivity throughout development. Furthermore, it is also important to include participants with autism across all IQ levels. While it is difficult to collect functional imaging data in the context of complex task performance in young children and individuals with low IQs, it is feasible to collect resting state fMRI data in these populations. It is important that future research determine to what degree underconnectivity applies to different age and IQ ranges within the autism population.

It will also be important for future research to explore the links between functional connectivity and diffusion tensor imaging measures of white matter integrity. Biologically realistic neural models also have great potential to examine the relationship between functional connectivity and anatomical connectivity (Horwitz et al., 2005).

\section{CONCLUSION}

Recent findings of atypical patterns in both functional and anatomical connectivity in autism have established that autism is a not a localized neurological disorder, but one that affects many parts of the brain in many types of thinking tasks. fMRI studies repeatedly find evidence of decreased coordination between frontal and posterior brain regions in autism, as measured by functional connectivity. Furthermore, neuroimaging studies have also shown evidence of an atypical pattern of frontal white matter development in autism. These findings indicate that limitations of brain connectivity give rise to the varied behavioral deficits found in autism. As research continues to explore these biological mechanisms, new intervention methods may be developed to help improve brain connectivity and overcome the behavioral impairments of autism.

\section{ACKNOWLEDGMENTS}

This work was supported by the Autism Centers of Excellence Grant (HD055748) from the National Institute of Child Health and Human Development; and by the National Institute of Mental Health (Training Grant T32MH019983-11 to Sarah E. Schipul).

mode sub-networks in autism spectrum disorder patients. Neuroimage 53, 247-256.

Au Duong, M. V., Audoin, B., Boulanouar, K., Ibarrola, D., Malikova, I., Confort-Gouny, S., Celsis, P., Pelletier, J., Cozzone, P. J., and Ranjeva, J. P. (2005). Altered functional connectivity related to white matter changes inside the working memory network at the very early stage of MS. J. Cereb. Blood Flow Metab. 25, 1245-1253.
Barnea-Goraly, N., Kwon, H., Menon, V., Eliez, S., Lotspeich, L., and Reiss, A. L. (2004). White matter structure in autism: preliminary evidence from diffusion tensor imaging. Biol. Psychiatry 55, 323-326.

Baron-Cohen, S., Leslie, A. M., and Frith, U. (1985). Does the autistic child have a "theory of mind"? Cognition 21, 37-46.

Barttfeld, P., Wicker, B., Cukier, S., Navarta, S., Lew, S., and Sigman, M. (2011). 
A big-world network in ASD: dynamical connectivity analysis reflects a deficit in long-range connections and an excess of short-range connections. Neuropsychologia 49, 254-263.

Ben Bashat, D., Kronfeld-Duenias, V., Zachor, D. A., Ekstein, P. M., Hendler, T., Tarrasch, R., Even, A., Levy, Y., and Ben Sira, L. (2007). Accelerated maturation of white matter in young children with autism: a high b value DWI study. Neuroimage 37, 40-47.

Büchel, C., Coull, J. T., and Friston, K. J. (1999). The predictive value of changes in effective connectivity for human learning. Science 283, 1538-1541.

Bullmore, E., and Sporns, O. (2009). Complex brain networks: graph theoretical analysis of structural and functional systems. Nat. Rev. Neurosci. 10, 186-198.

Carper, R. A., Moses, P., Tigue, Z. D., and Courchesne, E. (2002). Cerebral lobes in autism: early hyperplasia and abnormal age effects. Neuroimage 16, 1038-1051.

Casanova, M. F., Buxhoeveden, D. P., Switala, A. E., and Roy, E. (2002). Minicolumnar pathology in autism. Neurology 58, 428-432.

Castelli, F., Frith, C., Happé, F., and Frith, U. (2002). Autism, Asperger syndrome and brain mechanisms for the attribution of mental states to animated shapes. Brain 125, 1839-1849.

Cheng, Y., Chou, K. H., Chen, I. Y., Fan, Y. T., Decety, J., and Lin, C. P. (2010). Atypical development of white matter microstructure in adolescents with autism spectrum disorders. Neuroimage 50, 873-882.

Cherkassky, V. L., Kana, R. K., Keller, T. A., and Just, M. A. (2006). Functional connectivity in a baseline resting-state network in autism. Neuroreport 17 , 1687-1690.

Cheung, C., Chua, S. E., Cheung, V., Khong, P. L., Tai, K. S., Wong, T. K., Ho, T.P., and McAlonan, G. M. (2009). White matter fractional anisotrophy differences and correlates of diagnostic symptoms in autism. J. Child Psychol. Psychiatry 50, 1102-1112.

Coskun, M. A., Varghese, L., Reddoch, S., Castillo, E. M., Pearson, D. A., Loveland, K. A., Papanicolaou, A. C., and Sheth, B. R. (2009). Increased response variability in autistic brains? Neuroreport 20, 1543-1548.

Courchesne, E., Carper, R., and Akshoomoff, N. (2003). Evidence of brain overgrowth in the first year of life in autism. JAMA 290, 337-344.

Courchesne, E., Karns, C. M., Davis, H. R., Ziccardi, R., Carper, R. A., Tigue, Z. D., Chisum, H. J., Moses, P., Pierce, K., Lord, C., Lincoln, A. J., Pizzo, S., Schreibman, L., Haas, R. H., Akshoomoff, N. A., and Courchesne,
R. Y. (2001). Unusual brain growth patterns in early life in patients with autistic disorder: an MRI study. Neurology 57, 245-254.

Di Martino, A., Ross, K., Uddin, L. Q., Sklar, A. B., Castellanos, F. X., and Milham, M. P. (2009). Functional brain correlates of social and nonsocial processes in autism spectrum disorders: an activation likelihood estimation meta-analysis. Biol. Psychiatry 65, 63-74.

Fletcher, P. T., Whitaker, R. T., Tao, R., DuBray, M. B., Froehlich, A., Ravichandran, C., Alexander, A. L., Bigler, E. D., Lange, N., and Lainhart, J. E. (2010). Microstructural connectivity of the arcuate fasciculus in adolescents with high-functioning autism. Neuroimage 51, 1117-1125.

Frazier, T. W., and Hardan, A. Y. (2009). A meta-analysis of the corpus callosum in autism. Biol. Psychiatry 66, 935-941.

Friston, K. J. (1994). Functional and effective connectivity in neuroimaging: a synthesis. Hum. Brain Mapp. 2, 56-78.

Frith, U. (1989). Autism: Explaining the Enigma. Oxford: Blackwell.

Frith, U., and Happé, F. (1994). Language and communication in autistic disorders. Philos. Trans. R. Soc. Lond., B, Biol. Sci. 346, 97-104.

Gaffney, G. R., Kuperman, S., Tsai, L. Y., Minchin, S., and Hassanein, K. M. (1987). Midsagittal magnetic resonance imaging of autism. $\mathrm{Br}$. J. Psychiatry 151, 831-833.

Hardan, A. Y., Pabalan, M., Gupta, N., Bansal, R., Melhem, N. M., Fedorov, S., Keshavan, M. S., and Minshew, N. J. (2009). Corpus callosum volume in children with autism. Psychiatry Res. 174, 57-61.

Herbert, M. R., Ziegler, D. A., Makris, N., Filipek, P. A., Kemper, T. L., Normandin, J. J., Sanders, H. A., Kennedy, D. N., and Caviness, V. S. Jr. (2004). Localization of white matter volume increase in autism and developmental language disorder. Ann. Neurol. 55, 530-540.

Horwitz, B. (2003). The elusive concept of brain connectivity. Neuroimage 19, 466-470.

Horwitz, B., Rumsey, J. M., Grady, C. L., and Rapoport, S. I. (1988). The cerebral metabolic landscape in autism: intercorrelations of regional glucose utilization. Arch. Neurol. 45, 749-755.

Horwitz, B., Warner, B., Fitzer,J., Tagamets, M. A., Husain, F. T., and Long, T. W. (2005). Investigating the neural basis for functional and effective connectivity. Application to fMRI. Philos. Trans. R. Soc. Lond., B, Biol. Sci. 360, 1093-1108.
Jones, T. B., Bandettini, P. A., Kenworthy, L., Case, L. K., Milleville, S. C., Martin, A., and Birn, R. M. (2010). Sources of group differences in functional connectivity: an investigation applied to autism spectrum disorder. Neuroimage $49,401-414$.

Just, M. A., Cherkassky, V. L., Keller, T. A., Kana, R. K., and Minshew, N. J. (2007). Functional and anatomical cortical underconnectivity in autism: evidence from an FMRI study of an executive function task and corpus callosum morphometry. Cereb. Cortex 17 951-961.

Just, M. A., Cherkassky, V. L., Keller, T. A., and Minshew, N. J. (2004). Cortical activation and synchronization during sentence comprehension in high-functioning autism: evidence of underconnectivity. Brain 127, 1811-1821.

Kana, R. K., Keller, T. A., Cherkassky, V.L., Minshew, N. J., and Just, M.A. (2006). Sentence comprehension in autism: thinking in pictures with decreased functional connectivity. Brain 129, 2484-2493.

Kana, R. K., Keller, T. A., Cherkassky, V. L., Minshew, N. J., and Just, M. A. (2009). Atypical frontal-posterior synchronization of theory of mind regions in autism during mental state attribution. Soc. Neurosci. 4, 135-152.

Kana, R. K., Keller, T. A., Minshew, N. J., and Just, M. A. (2007). Inhibitory control in high-functioning autism: decreased activation and underconnectivity in inhibition networks. Biol. Psychiatry 62, 198-206.

Ke, X., Tang, T., Hong, S., Hang, Y., Zou, B., Li, H., Zhou, Z., Ruan, Z., Lu, Z., Tao, G., and Liu, Y. (2009). White matter impairments in autism, evidence from voxel-based morphometry and diffusion tensor imaging. Brain Res 1265, 171-177.

Keary, C. J., Minshew, N. J., Bansal, R., Goradia, D., Fedorov, S., Keshavan, M. S., and Hardan, A. Y. (2009). Corpus callosum volume and neurocognition in autism. J. Autism Dev. Disord. 39, 834-841.

Keller, T. A., and Just, M. A. (2009). Altering cortical connectivity: remediation-induced changes in the white matter of poor readers. Neuron 64, 624-631.

Keller, T. A., Kana, R. K., and Just, M. A. (2007). A developmental study of the structural integrity of white matter in autism. Neuroreport 18, 23-27.

Kennedy, D.P., and Courchesne,E. (2008). The intrinsic functional organization of the brain is altered in autism. Neuroimage 39, 1877-1885.

Kleinhans, N. M., Richards, T., Sterling, L., Stegbauer, K. C., Mahurin, R. Johnson, L. C., Greenson, J., Dawson, G., and Aylward, E. (2008). Abnormal functional connectivity in autism spectrum disorders during face processing. Brain 131, 1000-1012.

Koshino, H., Carpenter, P. A., Minshew, N. J., Cherkassky, V. L., Keller, T. A., and Just, M. A. (2005). Functional connectivity in an fMRI working memory task in high-functioning autism. Neuroimage 24, 810-821.

Koshino, H., Kana, R. K., Keller, T. A., Cherkassky, V. L., Minshew, N. J., and Just, M. A. (2008). fMRI investigation of working memory for faces in autism: visual coding and underconnectivity with frontal areas. Cereb. Cortex 18, 289-300.

Kumar, A., Sundaram, S. K., Sivaswamy, L., Behen, M. E., Makki, M. I., Ager, J., Janisse, J., Chugani, H. T., and Chugani, D. C. (2010). Alterations in frontal lobe tracts and corpus callosum in young children with autism spectrum disorder. Cereb. Cortex 20, 2103-2113.

Lee, J. E., Bigler, E. D., Alexander, A. L., Lazar, M., DuBray, M. B., Chung, M. K., Johnson, M., Morgan, J., Miller, J. N., McMahon, W. M., Lu, J., Jeong, E. K., and Lainhart,J.E. (2007). Diffusion tensor imaging of white matter in the superior temporal gyrus and temporal stem in autism. Neurosci. Lett. 424, 127-132.

Lombardo, M. V., Chakrabarti, B., Bullmore, E. T., Sadek, S. A., Pasco, G., Wheelwright, S. J., Suckling, J., and Baron-Cohen, S. (2010). Atypical neural self-representation in autism. Brain 133, 611-624.

Mason, R. A., Williams, D. L., Kana, R. K., Minshew, N., and Just, M. A. (2008). Theory of mind disruption and recruitment of the right hemisphere during narrative comprehension in autism. Neuropsychologia 46, 269-280.

Meyer-Lindenberg, A., Poline, J. B., Kohn, P. D., Holt, J. L., Egan, M. F., Weinberger, D. R., and Berman, K. F. (2001). Evidence for abnormal cortical functional connectivity during working memory in schizophrenia. Am. J. Psychiatry 158, 1809-1817.

Minshew, N. J., Goldstein, G., and Siegel, D. J. (1997). Neuropsychologic functioning in autism: profile of a complex information processing disorder. J. Int Neuropsychol. Soc. 3, 303-316.

Mizuno, A., Villalobos, M. E., Davies, M. M., Dahl, B. C., and Muller, R. A. (2006). Partially enhanced thalamocortical functional connectivity in autism. Brain Res. 1104, 160-174.

Monk, C. S., Peltier, S. J., Wiggins, J. L., Weng, S. J., Carrasco, M., Risi, S., and Lord, C. (2009). Abnormalities of intrinsic functional connectivity in autism spectrum disorders. Neuroimage 47, 764-772. 
Monk, C. S., Weng, S. J., Wiggins, J. L., Kurapati, N., Louro, H. M., Carrasco, M., Maslowsky, J., Risi, S., and Lord, C. (2010). Neural circuitry of emotional face processing in autism spectrum disorders. J. Psychiatry Neurosci. 35, 105-114.

Mostofsky, S. H., Powell, S. K., Simmonds, D. J., Goldberg, M. C., Caffo, B., and Pekar, J. J. (2009). Decreased connectivity and cerebellar activity in autism during motor task performance. Brain 132, 2413-2425.

Mottron, L., and Burack, J. (2001). "Enhanced perceptual functioning in the development of autism," in The Development of Autism: Perspectives from Theory and Research, eds J. A. Burack, T. Charman, N. Yirmiya, and P. R. Zelazo (Mahwah, NJ: Erlbaum), 131-148.

Mottron, L., Dawson, M., Soulieres, I., Hubert, B., and Burack, J. A. (2006). Enhanced perceptual functioning in autism: an update, and eight principles of autistic perception. J. Autism Dev. Disord. 36, 27-43.

Murias, M., Webb, S. J., Greenson, J., and Dawson, G. (2007). Resting state cortical connectivity reflected in EEG coherence in individuals with autism. Biol. Psychiatry 62, 270-273.

Noonan, S. K., Haist, F., and Muller, R. A. (2009). Aberrant functional connectivity in autism: evidence from lowfrequency BOLD signal fluctuations. Brain Res. 1262, 48-63.

Ozonoff, S., Pennington, B., and Rogers, S. J. (1991). Executive function deficits in high-functioning autistic individuals: relationship to theory of mind. J. Child Psychol. Psychiatry 32, 1081-1105.

Paakki, J. J., Rahko, J., Long, X., Moilanen, I., Tervonen, O., Nikkinen, J., Starck, T., Remes, J., Hurtig, T., Haapsamo, H., Jussila, K., Kuusikko-Gauffin, S., Mattila, M. L., Zang, Y., and Kiviniemi, V. (2010). Alterations in regional homogeneity of resting-state brain activity in autism spectrum disorders. Brain Res. 1321, 169-179.

Pugh, K. R., Mencl, W. E., Shaywitz, B. A., Shaywitz, S. E., Fulbright, R. K., Constable, R. T., Skudlarski, P., Marchione, K. E., Jenner, A. R., Fletcher, J. M., Liberman, A. M., Shankweiler, D. P., Katz, L., Lacadie, C., and Gore, J. C. (2000). The angular gyrus in developmental dyslexia: task-specific differences in functional connectivity within posterior cortex. Psychol. Sci. 11, 51-56.

Rice, S. A., Bigler, E. D., Cleavinger, H. B., Tate, D. F., Sayer, J., McMahon, W., Ozonoff, S., Lu, J., and Lainhart, J. E. (2005). Macrocephaly, corpus callosum morphology, and autism. J. Child Neurol. 20, 34-41.

Sahyoun, C. P., Belliveau, J. W., Soulieres, I., Schwartz, S., and Mody, M. (2010). Neuroimaging of the functional and structural networks underlying visuospatial vs. linguistic reasoning in highfunctioning autism. Neuropsychologia 48, 86-95.

Schipul, S. E., Williams, D. L., Keller, T. A., Kana, R. K., Minshew, N. J., and Just, M. A. (2008). "Learning to detect lies in autism," in Poster Presented at the International Meeting for Autism Research, London, UK.

Shukla, D. K., Keehn, B., and Muller, R. A. (2010). Regional homogeneity of fMRI time series in autism spectrum disorders. Neurosci. Lett. 476, 46-51.

Silk, T. J., Rinehart, N., Bradshaw, J. L., Tonge, B., Egan, G., O'Boyle, M.W., and Cunnington, R. (2006). Visuospatial processing and the function of prefrontal-parietal networks in autism spectrum disorders: a functional MRI study. Am. J. Psychiatry 163, 1440-1443.

Solomon, M., Ozonoff, S. J., Ursu, S., Ravizza, S., Cummings, N., Ly, S., and Carter, C. S. (2009). The neural substrates of cognitive control deficits in autism spectrum disorders. Neuropsychologia 47, 2515-2526.
Stanfield, A. C., McIntosh, A. M., Spencer, M. D., Philip, R., Gaur, S., and Lawrie, S.M. (2008). Towards a neuroanatomy of autism: a systematic review and meta-analysis of structural magnetic resonance imaging studies. Eur. Psychiatry 23, 289-299.

Sundaram, S. K., Kumar, A., Makki, M. I., Behen, M. E., Chugani, H. T., and Chugani, D. C. (2008). Diffusion tensor imaging of frontal lobe in autism spectrum disorder. Cereb. Cortex 18, 2659-2665.

Tepest, R., Jacobi, E., Gawronski, A., Krug, B., Moller-Hartmann, W., Lehnhardt, F. G., and Vogeley, K. (2010). Corpus callosum size in adults with highfunctioning autism and the relevance of gender. Psychiatry Res. 183, 38-43.

Thakkar, K. N., Polli, F. E., Joseph, R. M., Tuch, D. S., Hadjikhani, N., Barton, J. J., and Manoach, D. S. (2008). Response monitoring, repetitive behaviour and anterior cingulate abnormalities in autism spectrum disorders (ASD). Brain 131, 2464-2478.

Tian, L., Jiang, T., Wang, Y., Zang, Y., He, Y., Liang, M., Sui, M., Cao, Q., Hu, S., Peng, M., and Zhuo, Y. (2006). Altered resting-state functional connectivity patterns of anterior cingulate cortex in adolescents with attention deficit hyperactivity disorder. Neurosci. Lett. 400, 39-43.

Turner, K. C., Frost, L., Linsenbardt, D., McIlroy, J. R., and Müller, R. A. (2006). Atypically diffuse functional connectivity between caudate nuclei and cerebral cortex in autism. Behav. Brain Funct. 2, 34.

Vidal, C. N., Nicolson, R., DeVito, T. J. Hayashi, K. M., Geaga, J. A., Drost, D. J., Williamson, P. C., Rajakumar, N., Sui, Y., Dutton, R. A., Toga, A. W., and Thompson, P. M. (2006). Mapping corpus callosum deficits in autism: an index of aberrant cortical connectivity. Biol. Psychiatry 60, 218-225.
Villalobos, M. E., Mizuno, A., Dahl, B. C., Kemmotsu, N., and Muller, R. A. (2005). Reduced functional connectivity between $\mathrm{V} 1$ and inferior frontal cortex associated with visuomotor performance in autism. Neuroimage 25, 916-925.

Welchew, D. E., Ashwin, C., Berkouk, K., Salvador, R.,Suckling,J., Baron-Cohen, S., and Bullmore, E. (2005). Functional disconnectivity of the medial temporal lobe in Asperger's syndrome. Biol. Psychiatry 57, 991-998.

Weng, S. J., Wiggins, J. L., Peltier, S. J., Carrasco, M., Risi, S., Lord, C., and Monk, C. S. (2010). Alterations of resting state functional connectivity in the default network in adolescents with autism spectrum disorders. Brain Res. 1313, 202-214.

Wicker, B., Fonlupt, P., Hubert, B., Tardif, C., Gepner, B., and Deruelle, C. (2008). Abnormal cerebral effective connectivity during explicit emotional processing in adults with autism spectrum disorder. Soc. Cogn. Affect. Neurosci. 3, 135-143.

Conflict of Interest Statement: The authors declare that the research was conducted in the absence of any commercial or financial relationships that could be construed as a potential conflict of interest.

Received: 24 August 2010; accepted: 08 February 2011; published online: 22 February 2011.

Citation: Schipul SE, Keller TA and Just MA (2011) Inter-regional brain communication and its disturbance in autism. Front. Syst. Neurosci. 5:10. doi: 10.3389/ fnsys. 2011.00010

Copyright $\odot 2011$ Schipul, Keller and Just. This is an open-access article subject to an exclusive license agreement between the authors and Frontiers Media SA, which permits unrestricted use, distribution, and reproduction in any medium, provided the original authors and source are credited. 